\title{
Asesoramiento Colaborativo en Escuelas Municipales: Posicionamientos y Desafíos del Rol de Amigo Crítico en un Proyecto de Desarrollo Escolar Inclusivo
}

\section{Collaborative Counseling in Municipal Schools: Positions and Challenges of the Role of Critical Friend in an Inclusive School Development Project}

\author{
Jorge E. Soto * \\ Ignacio Figueroa \\ Cristopher Yáñez-Urbina \\ Universidad Diego Portales
}

\begin{abstract}
Este trabajo recoge una experiencia de asesoramiento realizada a un conjunto de escuelas de la Región Metropolitana de Chile, para el diseño e implementación de un plan de desarrollo inclusivo, a partir de la propuesta de la Guía para la Inclusión Educativa. Dicho plan buscó identificar y abordar barreras a la inclusión, detectadas por el colectivo y con el apoyo de un asesor externo denominado amigo crítico. El artículo puntualiza en la comprensión de este rol, como pieza importante de una estrategia de asesoramiento colaborativo. Por medio de un estudio de casos y un análisis cualitativo, se caracterizan los diversos posicionamientos y estrategias desplegadas por estos asesores, a partir de la identificación de dos ejes centrales: el del control/autonomía y según los focos reflexivo/instrumental. Los resultados permiten problematizar respecto de las condiciones, implicancias y desafíos para el trabajo de asesoramiento colaborativo, desde un enfoque inclusivo, en el contexto de la educación municipal.
\end{abstract}

Descriptores: Asesoramiento, Asistencia escolar, Innovación educativa, Aproximación participativa.

\begin{abstract}
This paper presents an experience of counseling in a group of schools from the Metropolitan Region of Chile for the design and implementation of an inclusive development plan, based on the proposal of the Index for Inclusion. This plan sought to identify and address barriers to inclusion, detected by the collective with the support of an external consultant called critical friend. The article points out in the understanding of this role, as an important piece of a collaborative counseling strategy. This study use a case study design with qualitative analysis, in order to identify the several positions and strategies deployed by these advisors, based on the identification of two central axes: control/autonomy and reflexive/instrumental focuses. The results problematize about the conditions, implications and challenges for collaborative counseling work, from an inclusive approach in the context of municipal education in Chile.
\end{abstract}

Keywords: Counseling, School attendance, Educational innovations, Participatory approach.

$\begin{array}{lll}\text { *Contacto: jorge.sotoc@mail.udp.cl } & \text { Recibido: } & 07 / 11 / 2016 \\ & 1^{a} \text { Evaluación: } & 18 / 01 / 2017 \\ \text { ISSN: 0718-7378 } & \text { Aceptado: } & 15 / 03 / 2017 \\ \text { www rinace net/rlei/ } & \end{array}$




\section{Introducción}

La necesidad de abordar las inequidades y desigualdades de nuestro sistema educativo ha determinado respuestas desde la política educativa a partir del principio de inclusión, el que se expresa en la no selección, el fin al copago y el lucro en la educación pública, conjuntamente con políticas de reconocimiento y enfoques curriculares que pretenden generar mejores respuestas del sistema educativo a la diversidad del alumnado.

Esta visión, que busca la reducción de la exclusión en la escuela, puede resultar bastante atractiva y en sintonía con transformaciones paradigmáticas, sin embargo, resulta aún antagónica al "mainstream" tradicional cuyos esquemas no suelen conversar con los ideales de una educación de inspiración democrática, abierta e inclusiva. Como señala Sapon-Shevin (2013) no podemos «cambiar las escuelas» sin observar las debilidades de nuestras políticas o al menos revisar aquellos valores que las inspiran, trascendiendo las estructuras tradicionales de los sistemas educativos.

Ciertamente, lo anterior demanda el desarrollo de capacidades pedagógicas, profesionales e institucionales para transformar los discursos, políticas y prácticas en el sistema y en nuestras escuelas, desde una perspectiva integral de desarrollo escolar inclusivo (Booth y Ainscow, 2012). A la vez que ahondar en la reflexión -en nuestro contexto- respecto de los procesos de cambio en las instituciones educativas desde un enfoque inclusivo, tanto como en el papel de las propuestas de apoyo a las escuelas, desde dicha perspectiva.

Aspectos relevantes en algunas propuestas de asesoramiento con enfoque inclusivo vendrían a ser el desplazamiento del énfasis en la implementación de políticas, hacia el desarrollo de capacidades para la identificación y levantamiento de barreras al aprendizaje y la participación en las propias comunidades educativas. Conjuntamente con una articulación consistente entre cuestiones de orden técnico-pedagógico con aspectos relativos al desarrollo de valores inclusivos. En esta línea, se han desarrollado propuestas bajo la formas de asesoramiento que orientan la elaboración de proyectos para propiciar la transformación integral de la escuela, y así volverla más receptiva, dialogante e inclusiva (Booth y Ainscow, 2012; Figueroa, Soto y Sciolla, 2016; Sales, Ferrández y Moliner, 2012).

En sintonía con lo anterior, entre los años 2014 y 2015 se desarrolló en una comuna de la región metropolitana de Santiago de Chile, una experiencia de asesoría para el trabajo en base a la Guía para la inclusión educativa (Booth y Ainscow, 2012), en el contexto de un programa de acompañamiento desarrollado por el Centro de Desarrollo Cognitivo (CDC) de la Universidad Diego Portales, con el apoyo de la red Index For Inclusion Network. Este programa de acompañamiento, basado en un enfoque de investigación acción, contempló la participación de asesores colaborativos: los amigos críticos. Estos asesores apoyaron a las escuelas y más específicamente a los equipos de planificación, a asumir los desafíos de cada uno de los procesos de planificación para el desarrollo inclusivo a partir de valores: la constitución de un equipo representativo, procesos de autoexploración, determinación de barreras al aprendizaje y la participación, y la definición de un plan de desarrollo inclusivo.

El presente artículo aborda el rol del amigo crítico (en adelante AC) en el contexto de una propuesta de asesoramiento colaborativo, como una dimensión relevante para favorecer procesos de apropiación del sentido transformador de la inclusión educativa 
(Figueroa, Soto y Sciolla, 2016). Específicamente, se propone comprender aspectos claves de su desenvolvimiento entre las características teóricamente prescritas para éste, desde el enfoque inclusivo, y las asumidas en la práctica en los contextos de asesoramiento escolar concretos, a fin de identificar condiciones y desafíos para el trabajo de asesoramiento colaborativo.

\section{Revisión de la literatura}

\subsection{La propuesta de la Guía para la inclusión educativa}

"La Guía para la inclusión educativa: desarrollando el aprendizaje y la participación en las escuelas" es un material inspirado en una concepción del desarrollo educativo y social estrechamente vinculado al valor de la participación democrática (Booth y Ainscow, 2012). De acuerdo a García y colaboradores (2013), la propuesta de La Guía encierra un sentido educativo y social definido como «crítico», ya que propone una transformación radical no solo de las escuelas, sino que del sistema educativo en su conjunto.

La Guía consiste en una serie de materiales para la autoevaluación integral de una escuela, invitando a dar coherencia a las mejoras bajo una variedad de etiquetas (educación en derechos, convivencia, educación ambiental, etc.) para orientarlas desde un enfoque inclusivo, fomentando el aprendizaje y la participación de todo la comunidad educativa (Booth y Ainscow, 2012).

Lo esencial de estos materiales es que proponen un abordaje de los procesos de inclusión a partir del conocimiento que posee la comunidad escolar acerca de su propia institución por medio de un proceso participativo, colaborativo y horizontal. El objetivo del trabajo implica explorar la institución educativa de forma abierta y compartida, planteando innovaciones transformacionales. El proceso se inicia con una fase de exploración de la escuela a través de indicadores y preguntas respecto de las culturas, políticas y prácticas, para luego determinar prioridades y planes de acción para levantar barreras en dichas dimensiones (Booth y Ainscow, 2012).

Respecto a experiencias con La Guía, se ha señalado que el cambio en la escuela desde una visión inclusiva es complejo y singular, no obstante el material resulta «sensible», dado que propende al debate y, en ocasiones, al conflicto, el que, sin embargo, puede ser superado dependiendo del abordaje de las escuelas (Durán et al., 2005). El mismo autor destaca la importancia de que la escuela se apropie de los materiales y no solo replique mecánicamente su propuesta técnica.

Por otra parte, un estudio sobre el uso de La Guía en 10 países europeos (Education, Audiovisual \& Culture Executive Agency, 2012) -Hungría, Países Bajos, Finlandia, Alemania, Bélgica, Austria, Irlanda, Portugal, Francia y Eslovenia- concluyó que los materiales contribuyeron a establecer prioridades en las escuelas, estructurando sus debates a partir del conocimiento de cómo se desarrollan los procesos de inclusión/exclusión en su comunidad. Se observó, así mismo, que la guía colaboró en configurar una visión clara y pertinente de metas y objetivos, permitiendo buscar posibilidades a través de la colaboración con otros organismos de la comunidad.

En tanto en el contexto nacional, un estudio de caso a partir del trabajo con La Guía para la inclusión en 7 escuelas municipales de una comuna de la Región Metropolitana, sostiene que para la apropiación, tanto del enfoque inclusivo como de las metodologías 
planteadas en dicho material, resultan claves 5 aspectos interrelacionados, a saber: el liderazgo inclusivo; una visión de la escuela como un todo; culturas colaborativas, un enfoque de investigación acción y el asesoramiento colaborativo a partir de la figura del AC (Figueroa, Soto y Sciolla, 2016).

En particular, sobre el AC, la propia Guía para la Inclusión Educativa (Booth y Ainscow, 2012) destaca su relevancia dentro de la propuesta y lo describe como un agente externo, quien apoya al grupo coordinador de la escuela en la investigación detallada de la misma, de modo sistemático y comprometido con el proceso, participando como un catalizador de procesos reflexivos y transformacionales.

No obstante, la importancia asignada al rol del AC, en la bibliografía que recoge experiencias con La guía para la inclusión, no se advierte un análisis detenido respecto del mismo en cuanto a las condiciones e implicancias de su práctica, y pareciera que existen variadas comprensiones sobre su función, dependiendo del tipo de proyecto, de su perfil y pertenencia institucional (Bozic, 2012; Carrington y Robinson, 2004; Duke, 2009; Figueroa y Muñoz, 2014; Figueroa, Soto y Sciolla, 2016; Hick, 2005; Lago, 2014; Nes, 2009; Oswald, 2010; Villalba, 2009). Al respecto, cabe abordar una caracterización más específica de este rol, pero no sin antes contextualizarlo brevemente en relación a los enfoques de asesoramiento escolar externo y el asesoramiento colaborativo en el que se enmarca.

\subsection{Asesoramiento colaborativo externo}

En los últimos años el enfoque de asesoramiento educativo externo ha evolucionado desde el modelo de servicios al de los programas de apoyo, instalándose la idea de que la escuela es la unidad básica de cambio y que los apoyos externos deben tener una orientación altamente contextual y flexible, que oriente el desarrollo de competencias en las escuelas, más que su suplantación (Segovia y Hernández, 2008). A nivel local, en la línea de la mejora escolar, las políticas educativas (Ley de Subvención Escolar Preferencial-SEP) han seguido esta dirección a partir de un "cambio de paradigma", pasando de entender a la escuela y los sostenedores como agentes pasivos que "reciben y acatan la política educativa”, a actores activos que deben construir sus demandas de apoyo conforme a un plan de mejora. Esta política manifiesta, además, que los principios que orientan la asistencia técnica externa deben ser el contribuir a dicha mejora y su sostenibilidad, mediante la instalación de capacidades y a partir de una visión integral del asesoramiento (Asesorías para el Desarrollo, 2014)

A pesar de este giro, cabe considerar dificultades típicas del apoyo escolar externo, tales como la posición marginal que ocupan en el contexto organizativo e interpersonal de las escuelas, respecto de los temas y conocimientos que son relevantes para las mismas; el carácter episódico y aislado de los apoyos en la ausencia de una estrategia articuladora, lo que se asocia a la redundancia de prestaciones. Mientras que, por otra parte, su imposición -cuando se da el caso- desde distintos niveles de la administración, sigue siendo un foco de resistencia para las comunidades escolares (Segovia y Hernández, 2008).

En particular, cabe mencionar que la asistencia externa con frecuencia deviene en acciones compensatorias respecto de la ausencia de recursos y capacidades, que las escuelas, por distintos motivos, no están en condiciones de cubrir y que, en ocasiones, son deliberadamente solicitadas para tales efectos (Asesorías para el Desarrollo, 2014). 
Consecuente con lo anterior, y a nivel nacional, se han reconocido una serie de inconsistencias entre el enfoque de sustentabilidad de la política de mejora y el perfil general que ha asumido la asistencia externa en la práctica. Esto se expresa en que son escasos los apoyos que se proponen instalar capacidades en la comunidad educativa para que asuman con autonomía los procesos de mejora continua y produzca un giro hacia el aprendizaje de todos los estudiantes de forma efectiva. Con todo, un aspecto valorado respecto de la asistencia técnica externa, tanto por las escuelas como por los sostenedores, ha resultado ser el aporte de una visión "desde afuera" de su quehacer, visión que definen como "más objetiva" y que - por su inmersión en la práctica- se les dificulta generar por sí mismas (Asesorías para el Desarrollo, 2014)

Respecto de la forma que asume esta función de observación de un asesor externo, se desprenden, a nuestro juicio, importantes implicancias relacionadas con la sostenibilidad y autonomía progresiva de las escuelas desde una perspectiva del desarrollo escolar inclusivo. En particular, como alternativa entre una hétero-observación y solución indicada permanentemente desde el exterior, y las auto-observaciones espurias o autocomplacientes de muchos auto diagnósticos institucionales, se sitúa la autobservación facilitada por una co-vision externa en el marco de un asesoramiento colaborativo.

Este asesoramiento colaborativo se puede entender por su oposición al modelo del asesoramiento como intervención, tal como lo plantea Escudero (1990) dicotomía que no obstante se puede degradar en una serie de posiciones según la propuesta de Nieto (1993, citado en Jiménez, 2005) la que es revisada en Nieto (2001) y que conviene tener en cuenta como marco de referencia para el análisis empírico del rol del AC, a saber:

- Intervención: el asesor como experto y autoridad técnica, motivado por intereses de rendimiento, productividad y control, con objetivos y procedimientos predeterminados, y en un modelo de acción lineal.

- Facilitador: el asesor como generador de un contexto de comunicación relevante y significativa, a partir de estrategias no directivas y motivadas en la generación de autonomía en el análisis y toma de decisiones.

- Colaboración Técnica: el asesor asume una posición horizontal con el /los asesorados y de corresponsabilidad en las acciones, pero con el foco en cuestiones de eficacia según criterios de mejora, predefinidos desde el exterior, y en cuarto lugar como

- Colaborador Crítico: un asesor que pone énfasis en relaciones horizontales, democráticas, en relaciona a la toma de decisiones, pero con un alto nivel de compromiso en torno de una corresponsabilidad transformativa y emancipatoria. El componente crítico aquí, hace alusión a la crítica ideológica o normativa

De los mencionados, los enfoques del asesor como facilitador y el de colaborador crítico, resultan ser formas de asesoramiento colaborativo que favorecerían procesos reflexivos y de aprendizaje organizacional (Anderson y Herr, 2007). No obstante, consideramos que estos enfoques tienen sustento en el supuesto -desde una perspectiva sistémica- que toda indicación externa requiere que esta sea inteligible desde dentro del sistema observado, en su propio contexto de operaciones y en el marco de sus posibilidades y 
expectativas (Dupuis, 2011). Asimismo, que una propuesta de cambio -altamente improbable- debe aspirar a ser construida y sostenida a partir de una lectura problematizada de la realidad, en este caso desde las propias comunidades escolares.

Desde la perspectiva del desarrollo escolar inclusivo, lo anterior redunda en que el proceso mismo de participación resulta un factor clave y un aspecto a auto observar, dado su efecto legitimador de los procesos de cambio, como en su catalización de aprendizaje colectivo en contextos de práctica, en la línea de aquellos factores que Ainscow (2004) define como impulsores fuertes del cambio, en tanto aportan a generar modificaciones significativas en la reflexión y en la práctica de las organizaciones escolares, destacando en ello la importancia de los procesos sociales de aprendizaje situado.

En este marco, es que la noción de AC cobra relevancia como pieza clave de una estrategia de asesoramiento en colaboración, y que resulta crítico en un doble sentido: en tanto facilitador reflexivo y en tanto movilizado por una visión crítica (normativa) a un cierto estado de cosas y comprometido en su transformación.

\subsection{Pero ¿qué es un amigo crítico?}

La noción de AC surge en el contexto educativo -aunque se encuentran antecedentes en el campo del desarrollo comunitario (Evans, 2014)- con aplicaciones en el liderazgo educativo (Loughran y Brubaker, 2015) y más frecuentemente como recurso reflexivo para el desarrollo profesional docente, el asesoramiento entre pares, análisis de datos, comunidades de aprendizaje, etc. (Escudero 2009; Foulger, 2010; Hargreaves y Fullan, 2014) y en esa misma línea, en la realización de los self study (Schuck y Russell, 2016). Su uso es frecuente en el campo del asesoramiento y la investigación acción con diversos focos, tales como el desarrollo curricular (Leite, 2002) la orientación (Calvo, Haya y Susinos 2010), la convivencia escolar (Ossa, Figueroa y Rodríguez, 2016) y la inclusión educativa (Bozic, 2012; Carrington y Robinson, 2004; Duke, 2009; Figueroa y Muñoz, 2014; Figueroa, Soto y Sciolla, 2016; Hick, 2005; Lago, 2014; Nes, 2009; Oswald; 2010; Villalba, 2009).

Aunque se encuentran variantes según las áreas específicas, el rol de AC lo podemos definir por constituirse en una relación de colaboración horizontal, entre dos o más personas, y cuyo fin es lograr una mejora de cierta práctica; en la comprensión de la misma e idealmente de los factores que la facilitan u obstaculizan. Su vehículo es el diálogo y las deliberaciones en torno a cuestiones tanto técnicas, de criterio y regulativas, sin excluir aspectos expresivos. Relación en la que una de las partes asume el rol de la crítica activa mientras que el otro el de procesarlas, sin obviar que se trata de una relación bidireccional en que toda crítica ha de poder ser justificada y o redefinida. A la base del rol está la búsqueda de armonización entre una posición de inmersión y compromiso del participante con una práctica, por un lado, y la distancia reflexiva del observador externo, por otro.

$\mathrm{El} \mathrm{AC}$ es frecuentemente descrito por su función especular (Calvo, Haya y Susinos, 2010; Escudero 2009) en su dimensión de crítica, en la medida en que ofrece contrapuntos, visiones alternativas, señalando puntos ciegos, ya sea frente a datos, posiciones o decisiones asumidas, ejerciendo en ese sentido de "abogado del diablo" (Anderson y Herr, 2007). Su posicionamiento no puede reclamar neutralidad, pues implica comprometerse activamente en el proceso de cambio (Kemmis y McTaggart, 1988; 
Stokes, 2003) a partir del establecimiento de un adecuado nivel de confianza y un razonable conocimiento del contexto de asesoramiento (Booth y Ainscow, 2012). El AC, junto a otra nominaciones afines (teórico crítico en Elliot, 1990) ha sido descrito a sí mismo por su carácter potenciador, en la medida en que su acción está orientada a promover el trabajo colaborativo, contribuir a una visión global de la escuela (Leite, 2002; Nieto, 2001), y sobre todo favorecer procesos reflexivos sobre las propias prácticas, explicitar supuestos y creencias que puedan facilitar la transformación de discursos y prácticas educativas (Vezub, 2007).

En el contexto específico de la investigación acción colaborativa, el rol del AC, cuyo asesoramiento discurre idealmente en la deliberación colectiva, resulta relevante en la medida en que: "cualquier tarea de investigación acción requiere un contexto social de intercambio, discusión y contrastación” (Contreras, 1994, p. 12). En tal sentido, además contribuye a los criterios de validez dialógica y catalítica, propuestas para la misma (Anderson y Herr, 2007).

\subsection{Contexto de asesoramiento}

La experiencia de asesoramiento que constituye el objeto de estudio, se desarrolló en 7 escuelas municipales de una comuna del sector norte de la Región Metropolitana de Chile. Su objetivo fue el diseño e implementación de un plan de desarrollo inclusivo, por parte de las comunidades escolares, a partir de la propuesta de la Guía para la Inclusión Educativa, y basada en un proceso de investigación acción.

Dicho proyecto surge por demanda del sostenedor municipal y en concordancia con los ejes de la política educativa comunal para la totalidad de las 7 escuelas de su administración, las cuales forman parte del programa SEP, el que otorga subvención a aquellos alumnos que se encuentran en situación de vulnerabilidad social

El proyecto de asesoramiento llevado a cabo incluyó una fase formativa inicial, ejecutada durante el segundo semestre del año 2014, que consistió en una formación colectiva en el conocimiento y uso de los materiales de la Guía para la Inclusión. La segunda etapa fue la conformación de los equipos de coordinación del proceso en cada escuela, inicialmente constituidos por distintos actores de la comunidad escolar (directivos, docentes, asistentes de la educación, apoderados y estudiantes), más el AC como asesor directo externo (3 amigos críticos distribuidos en las 7 escuelas). A este equipo le correspondió planificar y organizar el proceso indagatorio con la Guía. La tercera fase fue el proceso de autoexploración y definición de barreras a la inclusión y un plan de acción para su remoción. Estas dos fases se dieron durante el año 2015.

El rol definido en el proyecto para el AC, fue el de acompañar de forma continua el proceso en las comunidades educativas, buscando favorecer la apropiación de los materiales de la guía, colaborando en el proceso reflexivo de los equipos de coordinación de cada establecimiento.

\section{Método}

La investigación corresponde a un estudio de casos múltiple y desde un enfoque metodológico cualitativo. Entendiendo el estudio de caso aquí, como una estrategia de aproximación en profundidad y sin deslinde contextual a un fenómeno, desde una 
perspectiva naturalista (Kazez, 2009; Stake, 2007). Esta estrategia se ajusta al carácter comprensivo y situado de la investigación, desde un diseño etnográfico.

Los casos de estudio corresponden a las 7 escuelas que participaron en el proyecto de asesoramiento y la unidad de análisis se centró principalmente en las relaciones de asesoramiento entre el equipo coordinador del proyecto en la escuela (aproximadamente 7 miembros por escuela) y el AC. Los datos se produjeron a través de observación participante y notas de campo (91 notas) de las sesiones de asesoramiento registradas por los amigos críticos durante todo el período estudiado (año 2015); actas de las reuniones de coordinación y análisis del equipo asesor; y entrevistas a miembros de los equipos de las escuelas participantes (14 entrevistas).

Se realizó un análisis cualitativo de carácter tipológico (esquema cruzado), por medio de la generación y entrecruzamiento de categorías centrales, surgidas a partir de síntesis de codificación temática, vertical y cruzada de, y entre, los casos. La codificación buscó identificar modalidades típicas en las dinámicas de asesoramiento desarrolladas, a fin de delimitar y comprender sus modos y marcos de actuación durante el proceso.

\section{Resultados}

\subsection{Dos dimensiones del asesoramiento}

Se propone una organización de los resultados en función de dos dimensiones centrales respecto de los cuales las acciones los AC se pueden entender, según rasgos típicos. Ambas se describen brevemente, antes de pasar a ver su entrecruzamiento:

\subsubsection{Asesoramiento, según el modo autonomía/control: Colaboración Vs. intervención}

Si bien desde un punto de vista teórico el rol del AC se enmarca en el asesoramiento colaborativo, en la práctica se observan desplazamientos entre lo que se puede definir como dos polos extremos según el nivel de control, por una parte, centrada en la colaboración, y por otra centrada en la intervención.

Colaboración: La primera dimensión se caracteriza porque el rol de asesoramiento del AC no define ni los objetivos sustantivos, ni los resultados y estándares, como tampoco los recursos instrumentales de forma invariable, desde un criterio experto y externo. Más bien, esto viene a resultar de los procesos de co-definición de los propios participantes, su rol colaborador, es así apoyar en las propias definiciones y en los aspectos técnicos en su relación con un enfoque inclusivo tocante a los valores y a procedimientos consistentes técnica y normativamente. En este sentido, se caracteriza por propender a una distribución colegiada de la responsabilidad entre los participantes, en función de potenciar la autonomía. Cobra relieve aquí su posición de participante comprometido con el grupo, pero sin dejar de ser un externo

Intervención: Por otra parte, el asesor AC se acerca al polo o modelo de la intervención, cuando asume disposiciones y acciones directivas que centran en él responsabilidades referidas a la planificación, coordinación y activación del equipo, poniendo el foco en aspectos relacionados con un modelo de implementación, el cual registra algunos rasgos de dependencia externa del equipo, aunque no al extremo de predefinir objetivos y medios desde afuera. Se releva el carácter externo del asesor y se realizan presiones. 


\subsubsection{Asesoramiento según los focos del mismo o el qué: Lo reflexivo Vs. lo técnico-instrumental}

Cómo se ha dicho, una característica "esencial" del AC, dice relación con su rol potenciador de procesos de auto observación y reflexión con alta atención sobre el proceso. No obstante, en la práctica se observan, también, desplazamientos hacia un foco de acción de carácter técnico e instrumental, más operativo o centrado en los resultados.

Reflexividad-problematización: El AC, tal como lo prescribe su rol, se caracteriza por facilitar y mediar procesos reflexivos en torno a la autoexploración de la escuela y definiciones de acción para abordar barreras a la inclusión. De esta forma, el AC presenta ángulos, cuestionamientos y lecturas alternativas tanto a la producción y tratamiento de la información como a las decisiones adoptadas y sus conexiones con otras políticas e iniciativas de la escuela. Por otra parte, el AC busca tematizar y hacer observables los propios procedimientos llevados a cabo y su consistencia con el enfoque inclusivo, el nivel y tipo de participación de los distintos estamentos de la escuela en las acciones emprendidas, así como en los procedimientos adoptados en dicha lógica, es decir con foco en el cómo.

Lo técnico e instrumental: Esto refiere al foco del asesoramiento centrado en cuestiones de orden técnico-instrumental tales como apoyo respecto de competencias técnico metodológicas que los equipos de las escuelas suelen no poseer (análisis y procesamiento de datos, instrumentos de evaluación entre otros). Además, cumple una función en relación a las necesidades del tiempo, poniendo foco en aspectos operativos e instrumentales orientados al resultado del proyecto.

\subsection{Posiciones del rol de amigo crítico en la práctica de asesoramiento}

A partir del cruce de las dos dimensiones antes descritas, se configuraron cuatro posiciones que el AC asume en su función de asesor colaborativo, considerando las condiciones dadas y las que gestiona, en la concretización de su rol en la práctica. Cabe destacar que las 4 posiciones son virtualidades "ocupadas" por todos los asesores, según el momento, requerimientos y condiciones del contexto específico (figura 1).

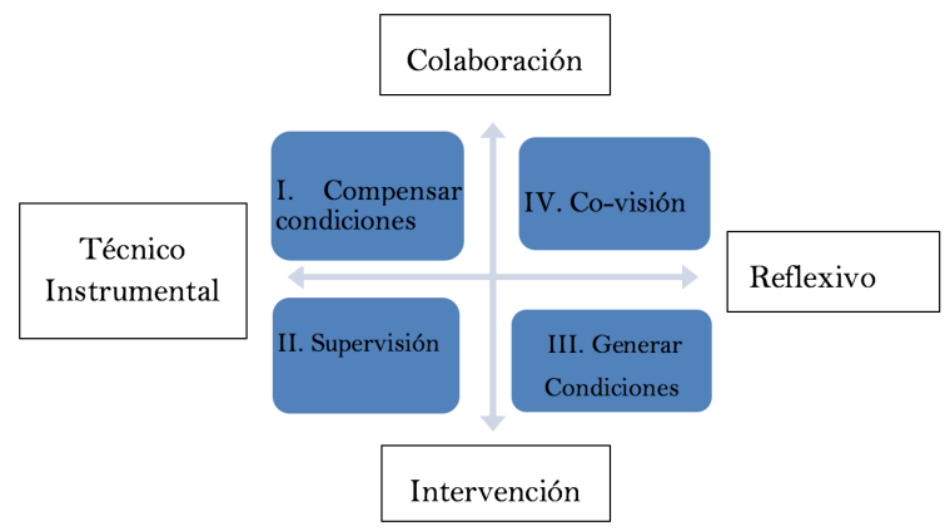

Figura 1. Cuadrante de posiciones según ejes del control (vertical) y el foco (horizontal) del asesoramiento.

Fuente: Elaboración propia.

\subsubsection{Compensar condiciones}

Esta surge cuando la acción del asesor se sitúa en la confluencia entre la colaboración y el foco en cuestiones en orden técnico, instrumental y operativo, orientadas a los 
resultados. Generalmente la colaboración en relación a esta posición se genera para compensar condiciones que la escuela "no posee", tales como habilidades técnicas específicas propias del proceso de investigación acción, falta de recursos humanos y/o de tiempo otorgado por la dirección a las actividades implicadas:

Juanita me muestra las encuestas que respondieron los apoderados, me ofrezco a tabularlas, claramente ella no sabe qué hacer con ellas. (Nota de campo 7, escuela 7)

Esta posición se puede entender en referencia a las figuras (metafóricas) contrapuestas del activista y del militante en la esfera de la acción política. Así el asesor activista es un participante-asesor que está compelido por cierto sentido de urgencia frente al avance del proyecto y de compromiso (eventualmente de culpa), pero desde una posición periférica respecto de los reales intereses de la escuela. Ello a partir de acciones puntuales cuyo riesgo principal es devenir en voluntarismo, dada la falta de articulación y sentido del proyecto y de su rol en la escuela. Así el asesor puede terminar "tirando" del proyecto, asumiendo la responsabilidad de sacarlo adelante (que no equivale a tener el control) comenzando siempre de nuevo y con el consiguiente desgaste:

\footnotetext{
El amigo crítico recuerda además que envió en el mes de septiembre una propuesta de contenidos y objetivos para la actividad con CEPA y subcentros, que aún no es contestada ni revisada por nadie de los integrantes del grupo. (Nota de campo 11, escuela 4)

Observo interés de parte de Felisa en este encuentro, siento que al inicio me devuelve la responsabilidad que tenemos los externos de 'apretar' a los establecimientos para responsabilizarse de sus compromisos, y hacerlos cumplir, pero de a poco va avanzando en reconocer el incesante trabajo en las escuelas y la fragmentariedad de las iniciativas que dificulta el que un colegio 'atienda con sentido' -y no solamente cumplir con la tarea- en la gestión de los organismos externos. (Nota de campo 5, escuela 3)
}

Por otra parte se da que esta posición, signada por la necesidad de brindar apoyo técnico y operativo, es ocupada por el AC desde un asesor-participante, como miembro del equipo que asume tareas técnicas e instrumentales, pero de forma orgánica para el logro de objetivos compartidos por el equipo coordinador. Lo anterior, por medio de una responsabilidad distribuida y cercana a la figura de un "militante", en el sentido de que colabora y coopera técnicamente en una configuración colectiva de las responsabilidades asumidas, aunque distante de un pensamiento único o de grupo.

Josefina ha traído también el resultado del trabajo de diagnóstico de los profesores,
son unas fichas resultado de trabajo en grupos. Me dice que por fortuna pudieron
terminar el trabajo. Le digo que es una muy buena base para seguir indagando en
los otros estamentos. Me ofrezco a ayudarla a sistematizar el resultado. Asumo que
este equipo está tan recargado de trabajo que una mano no les viene mal y que
además de esa forma puedo tener acceso directo a evidencias del proceso. Le
propongo que saquemos fotocopias a las fichas, son pocas. Le parece bien. De todos
modos, leemos en conjunto la evaluación que los profes han hecho de la actividad. Es
positiva en general, critican sí que se haya hecho la actividad en dos tandas. (Nota
de campo 6, escuela 7)
[El amigo crítico] ha sido como, así como bien responsable, adherente, no sé qué
palabra darle como para hablar de él porque en verdad fue como bien asertivo en
estas jornadas porque nos ayudó bastante. (Entrevista 1, escuela 4)

En ambos casos el compromiso y la responsabilidad se gestionan de forma diferente, en relación al foco instrumental y técnico de la ayuda, y la necesidad de compensación. 


\title{
3.2.2. Supervisión técnica-instrumental
}

Esta posición sitúa al asesoramiento entre el control del asesor (o intervención) y el foco en aspectos técnico instrumentales, típicamente en los resultados esperados, así como en la verificación de procedimientos (medios/fines). Es una posición propia del agente del sistema-entorno administrativo y enfatiza el carácter externo y experto del asesor. Siendo esta posición diametralmente contrapuesta al rol del asesoramiento colaborativo, el AC asume este modelo de acción en la práctica por 3 razones complementarias: las dos primeras por constricción estructural y la tercera como una estrategia del agente:

i) Constricciones desde "arriba" relacionadas con los tiempos de la administración a la que debe responder el asesor (el sostenedor municipal en este caso) en términos de la planificación, plazos, metas y resultados; situación que se agudiza frente a las contingencias propias de las escuelas (reuniones postergadas, urgencias del momento, paro docente etc.).

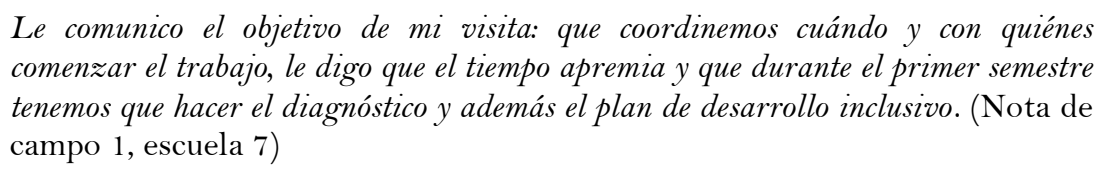

ii) Constricciones desde "abajo" que se configuran como expectativas de rol desde los equipos de las escuelas, mediadas por una cultura de la evaluación, supervisión externa y rendición de cuentas, y respecto de las cuales el asesor queda asociado por su relación con la administración y/o por su rol de "experto". Frente a ellas, él mismo asesor oscila entre su afirmación y su desmarque.

\begin{abstract}
Pregunto por el objetivo de una próxima reunión, para qué nos reuniremos... recuerdo que el objetivo de la reunión actual era elaborar material para los profesores, según acordamos la reunión pasada. Ver los avances, me dicen, pero los cuestionarios "no te los podemos tener toda la próxima semana", entendiendo que la reunión está terminando les digo que hagamos las cosas a su tiempo y que mi tarea no es supervisar los avances, sino acompañar y apoyar las decisiones de la escuela. La reunión termina en un clima que percibo incómodo, algo tenso. (Nota de campo 7 , escuela 1)
\end{abstract}

Porque a la larga si no estuviera los amigos críticos, yo creo que las encuestas recién las estaríamos haciendo. Porque a la larga nos va poniendo los límites y los tiempos, ya chiquillos, este es el momento de... ahí ella nos va poniendo los límites de los plazos y de las cosas que tenemos que hacer. Por ejemplo, ahora los insumos que le tenemos que entregar, que son las planificaciones y todo. (Entrevista 1, escuela 1)

iii) Como estrategia de presión para la armonización entre los polos de la colaboración reflexiva orientada al proceso y la colaboración técnico instrumental orientada a los resultados, por ejemplo, cuando la profundización en aquella no deja espacio a esta:

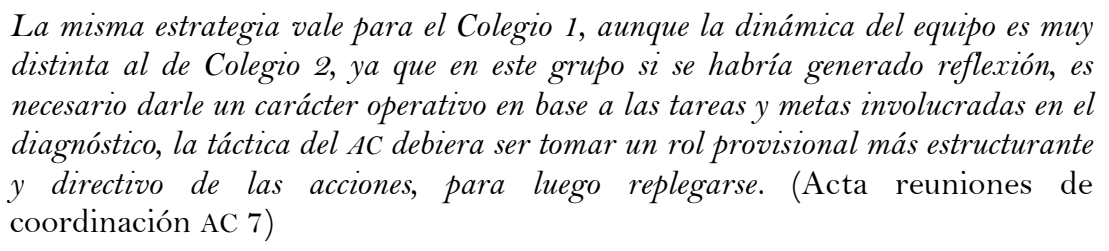
distinta al de Colegio 2, ya que en este grupo si se habría generado reflexión, es necesario darle un carácter operativo en base a las tareas y metas involucradas en el diagnóstico, la táctica del AC debiera ser tomar un rol provisional más estructurante $y$ directivo de las acciones, para luego replegarse. (Acta reuniones de coordinación AC 7)

En relación con lo anterior, tácticas puntuales de presión fueron el recurso a la comparación entre escuelas, presente en el marco de referencia de las mismas (principalmente las que se visualizan con mejores rendimientos) y aún cuando el sentido y la estrategia general de la propuesta de trabajo apunta a la colaboración interescuelas. 
Otras fueron presiones para maximizar (estirar) los escasos avances realizados, por ejemplo, en base a un diagnóstico incompleto, análisis parciales etc.

Sin duda, esta posición asume rasgos de una supervisión opuesta al asesoramiento colaborativo, la que en cierta medida resulta condicionada por el contexto y la imposición del mismo, entre otras. No obstante, esta posición puede ser modulada por el AC, es necesaria su consideración en profundidad en cuanto puede constituirse en un lugar de riesgo y "meta-barrera" para el asesoramiento colaborativo y el desarrollo escolar inclusivo.

\title{
3.2.3. Generación de condiciones
}

Esta posición surge en la conjunción entre un polo directivo desde AC y el foco en la reflexión/problematización sobre el proceso. Se despliegan aquí actuaciones que ejercen presión al equipo, en función de generar las condiciones básicas que requiere un proceso de trabajo bajo un enfoque inclusivo y orientado por valores, los que podríamos llamar “imperativos ético-procedimentales", tales como la participación, colaboración, diálogo igualitario, entre otros. Y de otra parte, condiciones como soportes institucionales (tiempos, instancias, articulación institucional de las iniciativas) y aspectos vinculados a la investigación acción, tales como el registro y la creación de memoria del proyecto: los acuerdos, decisiones, definiciones etc. Todos ellos, esenciales para visibilizar y proporcionar coherencia entre forma y contenido, proceso y resultado.

En este sentido el AC define que es necesario trabajar en ciertos prerrequisitos, de forma guiada, para desarrollar procesos de calidad. Trabaja para que se instalen y/o potencien recursos, más que compensarlos el mismo, y así favorecer las condiciones para un trabajo consistente con la propuesta:

\begin{abstract}
To voy haciendo algunas intervenciones de manera de lograr algunos objetivos que anticipamos junto al equipo coordinador: que podamos grabar las sesiones, conversar sobre la integración de todos los actores al equipo coordinador, organizar sesiones del equipo coordinador, detenernos a mirar y problematizar el avance en levantamiento de las opiniones y ver cómo seguimos explorando la inclusión en la escuela. (Nota de campo 7 escuela 2)

Vuelvo sobre el tema de los registros. Planteó la necesidad de que la escuela construya memoria de su propio proceso a partir de evidencias. Las actas son una forma de dejar memoria. Los registros fotográficos también. (Nota de campo 2, escuela 7)
\end{abstract}

Lo anterior se relaciona con la necesidad de generar auto-observación respecto de los propios procesos y muchas veces focalizar los medios como fines en sí mismos, es decir asumir un enfoque "procedimentalista" fuerte:

Reconozco el trabajo que se está haciendo, insisto en que también importa la manera de trabajar, no sólo los productos. (Nota de campo 7, escuela 1)

Una opción para ello es "instalar" una instancia de evaluación de proceso en los equipos, como forma de condicionar la autoobservación y tematización de aspectos procedimentales:

Otra de las observaciones importantes se refiere a la autoevaluación interna. Aprovecho de recordarles lo útil que los cuestionarios de autoevaluación serían para enriquecer esa parte: "El principal insumo para la evaluación interna es la reflexión del propio equipo a partir del cuestionario de autoevaluación individual que les envié por correo. La idea es que el propio equipo evalúe en proceso: ¿̇qué tan representativo fue el equipo? cómo se trabajó? ¿Cuáles fueron los obstáculos, las 
cosas que dificultaron el trabajo? Recordar que este enfoque, el proceso es tan relevante como el producto... (Nota de campo 15, escuela 1)

Por otra parte el AC realiza actuaciones en el plano de favorecer los procesos de participación sustantiva de los distintos actores, tanto en la composición como en las interacciones del equipo coordinador, así como en el levantamiento de información respecto de la escuela, su validación y legitimidad del proceso:

\begin{abstract}
Planteo la necesidad de integrar a todos los actores relevantes de la comunidad y la necesidad de que éstos sean representativos y ojalá elegidos por su propio equipo: apoderado, estudiante, dupla psicosocial, pedagogo teatral, equipo PIE. (Nota de campo 6, escuela 5)

Respecto de estos tres temas, el amigo crítico sugiere que se vuelva sistemática la evaluación de parte de los alumnos y que debiéramos hacia el final del proyecto volver a consultar a los alumnos si las acciones desarrolladas lograron mejorar los problemas que ellos detectaron. (Nota de campo 11, escuela 4)
\end{abstract}

Otra línea de acción del AC, dentro de estas coordenadas, pero apuntando a cuestiones de base material y organizativa, viene a definirse a partir de interpelaciones y propuestas de pautas de acción para generar sostén y salida a la propuesta, en escuelas en que el soporte institucional es débil o prácticamente ausente.

En síntesis, este posicionamiento del AC respecto de asumir un rol más directivo, si bien se distancia de su carácter colaborativo en su definición, se vuelca sobre cuestiones de fondo en la relación proceso/resultado con la idea de generar auto observaciones que avancen en problematizar según los requerimientos del enfoque inclusivo, en este sentido es una posición estratégica y constante del AC frente a la necesidad de generar dichas condiciones. Lo anterior, se muestra en la reflexiones del equipo asesor:

\begin{abstract}
Se visualizan avances, sin embargo, el rol del amigo crítico en ciertos momentos se ha caracterizado por un protagonismo, evaluado como necesario para aglutinar de las buenas intenciones de parte de los miembros de equipo. Queda como desafío el hecho de poder resituar ese rol de AC de modo progresivo dentro de la dinámica de acompañamiento colaborativo. (Acta reuniones de coordinación, AC 3).
\end{abstract}

Esta posición, no obstante, si no se ve acompañada de historia previa, prácticas informales y soportes institucionales de colaboración, puede devenir en presiones desde el exterior (y por tanto poco efectivas) muy asociadas a una colegialidad artificiosa (Hargreaves y Fullan 2014). Una alternativa sería en ese caso, una colegialidad guiada, es decir, mediante ciertos "toques", señales o arreglos institucionales propiciar que los actores opten por la colaboración como forma de trabajo.

\title{
3.2.4. Co-visión
}

Finalmente tenemos la posición que se ubica entre la colaboración como modalidad de interacción y la reflexión como foco de la misma. Posición que, como hemos visto, describe idealmente el espacio del AC, a partir de una gestión de la responsabilidad distribuida entre los participantes, y en función de potenciar la autonomía reflexiva y operativa de los equipos, así mismo en cuanto asesor no define ni objetivos sustantivos ni medios, alejándose del papel de experto externo y una posición de hegemonía epistemológica. Lo anterior, como rol de referencia del AC, se observa en la siguiente cita:

Esta fue la reunión en que se cumplió el rol de amigo crítico en observador más distante, la conversación y el proceso se fue dando por ellos mismos, con libertad pero de manera propositiva. (Nota de campo 13, escuela 6) 
Lo anterior, no obstante, en un plano de confianza necesario para el intercambio de críticas:

Claudia comenta entre risas que yo "le hice bolsa" el documento anterior y que ha estado trabajando en una nueva versión. Quiere que lo revisemos. (Nota de campo 15 , escuela 1)

Por otra parte, el foco privilegiado de dicha colaboración está puesto en la reflexión colectiva, tendiente a generar una visión problematizada de la realidad escolar a partir de los procesos de auto exploración y abordaje de barreras para la inclusión en la escuela. Básicamente, se trata de aportar a la construcción de visiones enriquecidas respecto de los problemas en torno a la inclusión, a partir de las estrategias de indagación, el análisis y establecimiento de relaciones (jerarquización y priorización) respecto de barreras y estrategias de acción a desarrollar:

A mí me sirvió, porque... como un espacio de reflexión para definir cuál era una barrera importante de trabajar, como fue el tema del pensamiento crítico. (Entrevista 1, escuela 1)

A pesar del cansancio, todos trabajaron muy bien en equipo y logramos construir objetivos que recogen y expresan las prioridades elegidas. Hicieron el ejercicio de establecer relaciones entre las barreras de cada sección, fueron vinculando las dimensiones entre sí y reflexionaron en torno a conceptos clave como ciudadanía, democracia y participación, generando una dinámica fluida y participativa. Los percibi muy interesados, particularmente a Paulina, que parecía estar muy interiorizada en el tema y enriqueció la discusión. (Nota de campo 18, Escuela 1)

La forma de favorecer dicha problematización consistió generalmente en acciones que frecuentemente referían a la focalización y la profundización en aspectos centrales, identificación de conexiones, el contrapunto o la visualización de aspectos no observados. lo anterior frente a una tendencia al abordaje episódico de los problemas, su identificación inmediata con acciones, el cierre prematuro de los mismos, o a particularizar sus causas en personas específicas o bien diluirlas en aspectos estructurales inabordables.

El rol de los amigos críticos siento yo que es un poco encauzar estas conversaciones, estos diálogos en torno a las temáticas de la inclusión. Ofrecer contrapuntos cuando nos vamos por las ramas, mostrarnos experiencias con la Guía, mostrarnos de alguna manera un contexto, apoyar, incentivar. (Entrevista 1, escuela 7)

(...) ella [amiga crítica] hizo que se provocara la reflexión acá, guió la reflexión, porque como que nos incentivó a decir por qué pensamos eso, qué podríamos hacer, era como siempre facilitando esta conversación. (Entrevista 2, escuela 5)

La búsqueda de profundización en torno a las barreras y sus conexiones, se aprecia en la siguiente secuencia de diálogo, tomada de una sesión de asesoría:

Equipo: Ellos [profesores] no quieren participar, porque están concentrados en la parte académica, quieren pensar en eso y no en otra cosa, y no tienen tiempo -dicen ellos- para hacer otras cosas (...) entonces ellos quieren solo dar cuenta de su trabajo, ese es el problema que tenemos

AC: ¿¿Eso es una barrera?

Equipo: de participación, sí, porque consideran que es un tiempo extra.

AC: Entonces, una alternativa puede ser ver cómo facilitar esa participación, no solo invitar a participar y tú ves cómo te las arreglas, como alivianarle horarios no lectivos, por ejemplo. 
Equipo: Es que eso ya se ha hecho, pero ellos dicen que esas horas las prefieren utilizar en otras cosas.

AC: Entonces ¿̇cuál es la barrera? si aun habiendo dotación de tiempo no quieren participar ¿qué impide que tengan una adhesión para participar y se identifiquen como colectivo, entonces?

Equipo: no sería el tiempo... (Transcripción de sesión, escuela 4)

Por otra parte destacamos que en esta posición el AC, en su ejercicio de plantear contrapuntos, suele constituirse en un agente irritador, en el sentido que el ejercicio de la crítica razonable, exige la justificación y/o revisión de ciertas decisiones o posturas, la puesta en tensión de repertorios de interpretación o predefiniciones: El desafío es sospechar, indagar sobre los fundamentos y la validez, develar la arbitrariedad o la mera coacción:

(...) Le pongo el tema a la directora sobre qué pasará si aparecen resultados de la aplicación de los cuestionarios que sean divergentes de lo que había optado inicialmente el equipo coordinador. (Nota de campo 4, escuela 2)

La acción del AC, además, busca articular las acciones emprendidas por el equipo con otras iniciativas desarrolladas por la escuela y principalmente con el proyecto institucional. Constantemente busca actualizar el sentido y enfoque del trabajo propuesto: Al leer el perfil de alumnos, docentes del PEI.... le sugiero que, por ejemplo, podemos ir haciendo relación entre lo que orienta y esperamos en el PEI con las acciones que estamos desarrollando en el proyecto inclusión. (Nota de campo 7, escuela 4)

El desarrollo de procesos de reflexión y problematización encontró, sin embargo, una serie de obstáculos respecto de las cuales hacerse cargo: Un primer tipo dice relación con la naturaleza de la instancia de interacción propiciada, cuando el diálogo reflexivo encuentra adecuados niveles de profundidad pero una persistente dificultad para encontrar cauces de acción, o bien cuando permanentemente asume un carácter catártico.

Por otra parte existen obstáculos en forma de restricciones a la comunicación en la forma de coacción a los participantes (parcial o total) o mediante la forma de una comunicación sistemáticamente distorsionada (manipulación/apariencia) ambas relacionadas con cuestiones de micro política.

No obstante, este posicionamiento del AC, encuentra su sentido en la medida en que se favorece también procesos de autonomía:
para mi la labor de ellos ha sido fundamental como te digo, sino estaríamos todavía ahí empezando a dar los primeros pasos y no estaríamos ya entre comillas caminando un poco más seguro nosotros. (Entrevista 1, escuela 3)

\section{Conclusiones}

El análisis de una propuesta concreta de asesoramiento colaborativo, con enfoque inclusivo, y centrado en la figura del amigo crítico, nos ha permitido identificar, más allá de sus definiciones teóricas y prescripciones normativas, una multiplicidad de marcos de actuación -algunos a priori antagónicos- respecto de los cuales este se ve sujetado y tensionado en su desempeño de rol. Lo anterior, creemos, aporta algunas distinciones útiles para abordar la complejidad de los procesos de cambio en los contextos estudiados, a la vez que plantea algunos desafíos. 
Si bien resulta necesaria y deseable la activación de genuinos procesos de reflexión colaborativa para la problematización de la escuela, a fin de levantar barreras a la inclusión, cabe señalar que la instalación y desarrollo de estas dinámicas reflexivas no es lineal, siendo más bien de carácter frágil, localizada y discontinua. La identificación del tipo de condiciones que afectarían dicho proceso fueron puestas en relación con los distintos posicionamientos y estrategias adoptados por los asesores.

Así, mientras que por una parte, dificultades propias a la reflexión colaborativa resultaron progresiva y relativamente abordables mediante estrategias dialógicas (focalización, conexión, justificación, etc.) en el plano mismo de la comunicación; otros obstáculos que se identificaron en el exterior a la misma (coacción, falta de soporte institucional y de participación) demandaron un posicionamiento de carácter directivo, el que fue desde el hacer ver dichas debilidades, hasta presionar para la generación de condiciones, muchas de ellas mínimas para esta actividad. Lo anterior es consistente con la propuesta de asesoramiento, en cuanto tematiza los valores procedimentales que busca alcanzar, sin embargo tiene sus límites, frente a la conformación de una colegialidad aparente o artificiosa (Hargreaves y Fullan, 2014).

Por otra parte, cuando el foco del asesoramiento resultó estar en los aspectos técnicos y operativos del mismo proyecto, la debilidades, relativas, en las capacidades de las escuelas, llamaron al asesor a un posicionamiento que osciló entre la compensación voluntarista -ausencia de autonomía y de control- y la cooperación técnica/instrumental, con una mayor conexión con el sentido del trabajo. En contraposición, pero en el mismo foco de asesoramiento, la externalidad del asesor, su relación con el sostenedor, el carácter impuesto y los marcos de referencia tradicionales de la escuela basados en el control y la rendición de cuentas- vinieron a colocar al amigo crítico (y este a colocarse) en la lógica de la supervisión, ejerciendo presión y enfrentado a una colección de "evidencias" a veces sin mayor trasfondo.

Dadas las condiciones señaladas, cabe decir que las posiciones descritas no son absolutas, es decir que, para un mismo asesor, estas variarán según el contexto y el momento del asesoramiento. Mientras que tampoco es dable reconocer una transición lineal entre las mismas. Sin duda, se desprende de lo anterior un desafío para el rol de amigo crítico, a saber, el poder reconocer estratégicamente su carácter móvil -podríamos decir liminal- y afrontar la necesidad de renegociación constante de su rol con la comunidad asesorada. Ello, a fin de evadir la trampa de quedar fijado en algo que no es o en su afirmación como algo que (aún) no puede ser.

En un plano más general, lo anterior invita a considerar lo complejo de buscar cambios en las instituciones a partir de valores inclusivos (Booth y Ainscow, 2012), confrontados, muchas veces, con las políticas promovidas por el propio sistema y las "formas de pensamiento útil” (Villarruel, 2012). Allí surgen tensiones típicas que enfrentan las comunidades escolares, y respecto de las cuales las estrategias de asesoramiento deben posicionarse: ¿Proceso o producto? ¿Colaboración o competencia? ¿Diversificación o estandarización? No resulta extraño, entonces, que interpelaciones de distinto signo resulten a veces poco comprensibles, o que frente a la progresiva intensificación del trabajo docente, la presión por resultados (Hargreaves, 1994) y las dificultades heredadas para abordar la diversidad, estas tensiones tomen formas de dicotomías irreconciliables. 
Cabe entonces, que las instituciones desarrollen procesos reflexivos o de "metacognición institucional” (Ossa, Figueroa y Rodríguez, 2016) y posicionamientos ético políticos para procesar, al menos parcialmente, las disarmonías y disparidades de nuestra política educativa. En ese sentido, uno de los desafíos que se plantean es la búsqueda necesaria de formatos de asesoramiento pertinentes para abordar consistentemente aspectos de la agenda inclusiva prescrita por la nueva política educativa chilena. En relación con ello, es que pueden ser útiles las reflexiones aquí planteadas respecto de una experiencia de asesoramiento colaborativo y el desenvolvimiento una de sus piezas claves, el amigo crítico.

\section{Agradecimientos}

Los autores agradecen a las comunidades educativas de la comuna de Huechuraba su colaboración y compromiso con el proyecto desarrollado. Asimismo, agradecen a los colegas que trabajaron en el programa de asesoramiento del Centro de Desarrollo Cognitivo y, muy especialmente, al profesor Tony Booth su empuje y acompañamiento en este proceso.

\section{Referencias}

Ainscow, M. (2004). El desarrollo de sistemas educativos inclusivos: ¿Cuáles son las palancas de cambio? Journal of Educational Change, 5(4), 1-20

Anderson, G. y Herr, K. (2007). El docente-investigador: Investigación-acción como una forma válida de generación de conocimientos. En I. Sverdlik (Ed.), La investigación educativa: Una herramienta de conocimiento y de acción (pp. 47-70). Buenos Aires: Noveduc

Asesorías Para el Desarrollo. (2014). Estudio sobre el funcionamiento de los servicios de Asistencia Técnica Educativa (ATE) chileno: Informe final. Santiago: MINEDUC y PNUD.

Booth, T. y Ainscow, M. (2012). Guía para la inclusión educativa: desarrollando el aprendizaje y la participación en las escuelas. Santiago: CSIE-FCF.

Bozic, A. (2012). Good practices and lessons learned in the application of the "Index for inclusion" methodology in elementary schools in Republika Srpska, Bosnia and Herzegovina. Sarajevo: Save the Children. doi:10.13140/2.1.3745.3609

Calvo, A., Haya, I. y Susinos T. (2012). El rol del orientador en la mejora escolar. Una investigación centrada en la voz del alumnado como elemento de cambio. Revista de Investigación en Educación, 10(2), 2012, 7-20.

Carrington, S. y Robinson, R. (2004). A case study of inclusive school development: A journey of learning. The International Journal of Inclusive Education, 8(2), 141-153. doi: $10.1080 / 1360311032000158024$.

Contreras, J. (1994). La investigación en la acción. Cuadernos de Pedagogía, 224, 7-12.

Duke, J. (2009). The use of the index for inclusion in a regional educational learning community. QUT Digital Repository. Recuperado de http://eprints.qut.edu.au/29400/

Dupuis, H. (2011). La teoría de la intervención como escenario de observación de las distinciones de la teoría de la evolución. Revista Mad - Universidad de Chile, 25, 34-42

Durán, D., Echeita, G., Giné, C., Miquel, E., Ruiz, C. y Sandoval, M. (2005). Primeras experiencias de uso de la guía para la evaluación y mejora de la educación inclusiva en el 
Estado Español. REICE. Revista Iberoamericana sobre Calidad, Eficacia y Cambio en Educación, 3(1), 464-467.

Education, Audiovisual \& Culture Executive Agency. (2012). Analysis of the use and value of the index for inclusion (Booth $\mathcal{O}^{\circ}$ Ainscow, 2011) and other instruments to assess and develop inclusive education practice in P2I partner countries. Bruselas: Fontys.

Elliot, J. (1990). La investigación acción en educación. Barcelona: Morata.

Escudero, J. (1990). ¿Qué es eso de intervención educativa? en Universidad de Murcia, Instituto de Ciencias de la Educación (Ed.), Intervencion en educación especial. pp.15-30, España.

Escudero, J. (2009). El amigo crítico, una posibilidad para la formación del profesorado en los centros. Compartim. Revista de Formació del Professorat, 4, 1-4.

Evans, S. (2014). The community psychologist as critical friend: promoting critical community praxis. Journal of Community $\&$ Applied Social Psychology, 25(4), 355-368. doi: $10.1002 /$ casp.2213

Figueroa, I. y Muñoz, Y. (2014). La guía para la inclusión educativa como herramienta de autoevaluación institucional: Reporte de una experiencia. Revista Latinoamericana de Educación Inclusiva, 8(2), 179-198.

Figueroa, I., Soto, J. y Sciolla, B. (2016). Dinámicas de recepción, apropiación y contextualización del enfoque de la guía para la inclusión educativa en escuelas municipales de una comuna de la Región Metropolitana. Pensamiento Educativo: Revista de Investigación Educacional Latinoamericana, 53(1), 1-13. doi: 10.7764/PEL.53.1.2016.4.

Foulger, T. (2010). External conversations an unexpected discovery about the critical friend in action research inquiries. Action Research, 8(2), 135-152. doi: 10.1177/1476750309351354

García, I., Romero, S., Aguilar, L., Lomelí, K. y Rodríguez, D. (2013). Terminología internacional sobre la educación inclusiva. Actualidades Investigativas en Educación, 13(1), 1-29. doi: $10.15517 /$ aie.v 13 i 1.11712

Hargreaves, A. y Fullan, M. (2014). Capital profesional. Madrid: Morata.

Hick, P. (2005). Supporting the development of more inclusive practices using the index for inclusion. Educational Psychology in Practice, 21(2), 117-122. doi: $10.1080 / 02667360500128754$

Jiménez, M (2005). Asesoramiento como investigación acción de segundo orden. Revista de Ciencias de la Educación, 12, 93-104.

Kazez, R. (2009). Los estudios de casos y el problema de la selección de la muestra: aportes del sistema de matrices de datos. Subjetividad y Procesos Cognitivos, 13(1), 71-89.

Kemmis, S. y McTaggart, R. (1988). Cómo planificar la investigación-acción. Madrid: Laertes.

Lago, M. (2014). Index para a inclusão: Uma possibilidade de intervenção institucional (Tesis doctoral). Universidad Federal de Río de Janeiro.

Leite, C. (2002). A figura do amigo crítico no assessoramento /desenvolvimiento de escolas curricularmente inteligentes. En M. Fernandes, J. Gonçalvez, M. Bolina, T. Salvado y T. Vitorino (Coords.), O particular e o global no virar do milenio (pp. 95-100). Lisboa: Ediciones Colibrí.

Loughran, J. y Brubaker, N. (2015). Working with a critical friend: A self-study of executive coaching. Studying Teacher Education, 11(3), 255-271. doi: 10.1080/17425964.2015.1078786 
Nes, K. (2009). The role of the index for inclusion in supporting school development in Norway: A comparative perspective. Research in Comparative and International Education, 4(3), 305320. doi: 10.2304/rcie.2009.4.3.305.

Nieto, J. (2001). Modelos de asesoramiento a organizaciones educativas. En J. Domingo (Coord.), Asesoramiento al centro educativo, colaboración y cambio en la institución (pp. 147-166). Barcelona: Octaedro-EUB.

Ossa, C., Figueroa, I. y Rodríguez, F. (2016). La metacognición institucional como herramienta para mejorar la gestión de la convivencia escolar. Actualidades Investigativas en Educación, 16(3), 1-18. doi: 10.15517/aie.v16i3.25962.

Oswald, M. (2010). Teacher learning during the implementation of the index for inclusion in a primary school (Tesis doctoral). Stellenboch University.

Sales, M. A., Fernández, R. y Moliner, O. (2012). Escuela intercultural inclusiva: estudio de caso sobre procesos de autoevaluación. Revista de Educación, 358, 153-173. doi: 10-4438/1988592X-RE-2012-358-187.

Sapon-Shevin, M. (2013). La inclusión real: una perspectiva de justicia social. Revista de Investigación en Educación, 1 1(3), 71-85.

Schuck, S. y Russell, T. (2016). Self-Study, Amistad crítica y las complejidades en la formación de profesores. En T. Rusell, R. Fuentealba y C. Hirmas (Comps.), Formadores de formadores, descubriendo la propia voz a partir del self study (pp. 117-132). Santiago: OEI.

Segovia, J. y Hernández V. (2008). Una mirada crítica a la función asesora desde los servicios de apoyo externo. Recorrido y prospectiva. Olhar de professor, 11(2), 63-80. doi: 10.5212/OlharProfr.v.11i1.063080

Stake, R. (2007). Investigación con estudio de casos. Madrid: Morata.

Stokes, L. (2003). Lecciones de una escuela indagadora: formas de indagación y condiciones para el aprendizaje del profesorado. En A. Lieberman y L. Miller (Eds.), La indagación como base de la formación del profesorado y la mejora de la educación (pp. 173-192). Barcelona: Octaedro.

Vezub, L. F. (2007). La formación y el desarrollo profesional docente frente a los nuevos desafíos de la escolaridad. Profesorado: Revista de Curriculum y Formación del Profesorado, 11(1), 23. http://doi.org/http://www.ugr.es/local/recfpro/rev111ART2.pdf

Villalba, M. (2009). Implementación del Indice de Inclusión desde la perspectiva de los alumnos (Tesis de maestría). Universidad de Cádiz.

Villarruel, M. (2012). Entornos políticos y dilemas sociales: los horizontes de la educación inclusiva. Perspectiva Educacional, 51(2), 4-20.

\section{Breve CV de los autores}

\section{Jorge Eduardo Soto Cárcamo}

Sociólogo, Máster en Investigación en Sociología Aplicada, Universidad Autónoma de Barcelona. Actualmente es coordinador de titulación y docente en el área de metodología del Magister en Desarrollo Cognitivo del Centro de Desarrollo Cognitivo de la Universidad Diego Portales. Posee experiencia en investigación, consultoría y asesoramiento en temáticas de educación intercultural e inclusión educativa. ORCID ID: 0000-0001-5107-5668. Email: jorge.sotoc@mail.udp.cl 


\section{Ignacio Figueroa Céspedes}

Psicólogo Educacional y Magister en Desarrollo Cognitivo. Actualmente coordina el área de Educación Continua del Centro de Desarrollo Cognitivo de la Universidad Diego Portales. Docente de la Carrera de Educación Diferencial y del Magister en Desarrollo Cognitivo de la UDP. Posee experiencia profesional en el ámbito de la Inclusión Educativa, el Desarrollo Cognitivo y la Convivencia Escolar; desarrollando líneas investigativas ligadas al Cambio Escolar y la Innovación Docente, desde un enfoque inclusivo y desarrollador. Miembro de la Red Index For Inclusion Network. ORCID ID: 0000-0002-2756-1831. Email: ignacio.figueroa@mail.udp.cl

\section{Cristopher Yáñez-Urbina}

Psicólogo de la Universidad de Santiago de Chile y cursa el Magister en Comunicación Política de la Universidad de Chile. Investigador responsable del proyecto Fondart línea Investigación Audiovisual, y ayudante de investigación en proyecto Fondecyt de iniciación ${ }^{\circ}{ }^{\circ} 115443$. Posee experiencia en temáticas de inclusión educativa y la comunicación. Actualmente es docente del Programa de Desarrollo de Habilidades Cognitivas y Socioemocionales de la Universidad Iberoamericana de Ciencias y Tecnologías, y del área de Formación Integral de la Escuela de Psicología de la Universidad de Santiago de Chile. ORCID ID: 0000-0001-7171-9686. Email: cristopher.urbina@usach.cl 